\title{
Fabrication and spectroscopy of erbium doped gallium lanthanum sulphide glass fibres for mid-infrared laser applications
}

\author{
T. Schweizer, D.J. Brady and D.W. Hewak \\ The Optoelectronics Research Centre, University of Southampton, UK, SO17 1BJ
}

Gallium lanthanum sulphide based glasses are proposed as high quality hosts for rare-earth doped, mid-infrared fibre lasers, that would offer compact and rugged sources for gas sensing, atmospheric transmission, and medical applications. The infrared emission spectroscopy of erbium doped glasses and fibres shows the potential of this glass host for the above applications. Mid-infrared transitions at 2.0, 2.75, 3.6, and $4.5 \mu \mathrm{m}$ have been detected and characterized.

\section{Introduction}

There is considerable international interest in developing mid-infrared (MIR) laser sources for gas sensing, atmospheric transmission, and medical applications. Many characteristic absorption 'fingerprints' of gases and vapours lie in the $2-5 \mu \mathrm{m}$ wavelength range, as do the atmospheric transmission windows. Our work targets specific wavelengths for these applications such as $4.3 \mu \mathrm{m}$ emission from $\mathrm{Dy}^{3+}$ for $\mathrm{CO}_{2}$ detection, $4.7 \mu \mathrm{m}$ from $\mathrm{Pr}^{3+}$ and $4.8 \mu \mathrm{m}$ from $\mathrm{Tb}^{3+}$ for $\mathrm{CO}$ and $\mathrm{O}_{3}$ detection, $3.4 \mu \mathrm{m}$ from $\mathrm{Pr}^{3+}$ for $\mathrm{CH}_{4}$ detection, and $3.9 \mu \mathrm{m}$ emission from $\mathrm{Ho}^{3+}$ for atmospheric transmission. Diode pumped rare-earth doped fibre lasers would offer compact and efficient

alternatives to the either relatively weak, or very complex MIR sources currently available, such as thermal emitters, gas lasers, OPO's or semiconductor lasers.

A fibre laser geometry is advantageous as the waveguiding gives good overlap of pump and laser modes, and the pump intensity is high, reducing the threshold, and increasing efficiency. A prerequisite for the rare-earth host material is a low phonon energy leading to MIR transparency and low non-radiative decay rates and therefore high quantum efficiency of radiative transitions. Conventional silica fibres do not fulfill these requirements leading to a need for new host materials with lower phonon energies that must also be suitable for fibre drawing. Mid-infrared transmitting fibres are currently either fluorozirconate based, with the disadvantages of being hygroscopic, and having a relatively small transmission window $(\sim 1-3 \mu \mathrm{m})$, or $\mathrm{As}_{2} \mathrm{X}_{3}(\mathrm{X}=\mathrm{S}$, Se etc.) based with the disadvantage of toxicity should the fibre burn or degrade, and low rare-earth solubility. Our approach to MIR lasers is based on stable, non-toxic, and non-hygroscopic chalcogenide glasses based on gallium and lanthanum sulphides with the molar composition $70 \mathrm{Ga}_{2} \mathrm{~S}_{3}: 30 \mathrm{La}_{2} \mathrm{~S}_{3}$ (GLS), and $70 \mathrm{Ga}_{2} \mathrm{~S}_{3}: 30 \mathrm{La}_{2} \mathrm{O}_{3}$ (GLSO), both of which have been drawn into fibre successfully. [1] They have a low phonon energy (425 $\mathrm{cm}^{-1}$ for GLS) and a wide transmission window that extends to beyond $8 \mu \mathrm{m}$ with an absolute loss minimum around $4 \mu \mathrm{m}$.

\section{Glass properties.}

Starting materials of $\mathrm{Ga}_{2} \mathrm{~S}_{3}, \mathrm{La}_{2} \mathrm{~S}_{3}$, or $\mathrm{La}_{2} \mathrm{O}_{3}$ powders are batched and mixed, before transferring to a vitreous carbon crucible in a silica tube. Melting is done at $1150^{\circ} \mathrm{C}$ for sever- al hours to give a homogenous glass ingot upon quenching. For thermal analysis $30 \mathrm{~g}$ test melts are produced, which also give enough glass for small bulk glass samples to be cut and polished from. These are used for spectroscopic measurements. Fibre drawing is done by a rod-in-tube method, for which $220 \mathrm{~g}$ ingots of glass are produced, cut, and optically polished into rods and tubes. Fig. 1 shows a glass ingot, some polished tubes, and a tube that has been stretched into a cane for insertion into a tube. 


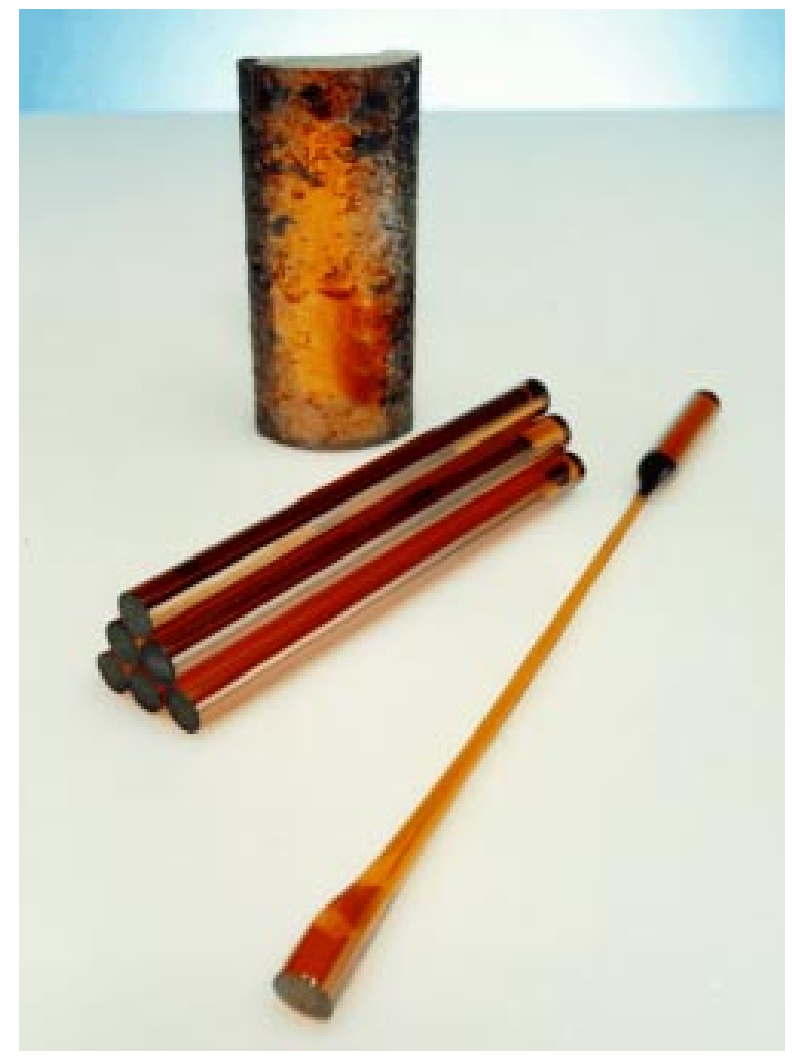

Fig. 1. A photograph of GLS glass; a 220g ingot, some polished rods (110mm x 8mm), and a drawn 'cane'.

Thermal analysis is performed using a Perkin-Elmer DTA7 for transition temperatures, and for viscosity measurements a Perkin Elmer TMA7 is used, which has been adapted for parallel plate rheometry. It is best to perform fibre drawing at temperatures well below the crystallisation temperature of the glass. To this end a simple test of the thermal quality of a glass with respect to fibre drawing may be measured by the separation of the drawing temperature $\left(T_{\eta}\right.$; the temperature at which the viscosity $\sim 10^{6}$ poise) and the peak crystallisation temperature $\left(T_{p}\right)$. A large value for $T_{p^{-}} T_{\eta}$ would give a glass that is more suitable for fibre drawing. For GLS $T_{p^{-}} T_{\eta}=46^{\circ} \mathrm{C}$, and for GLSO $T_{p}-T_{\eta}=130^{\circ} \mathrm{C}$. This makes GLSO an easier glass to draw into fibre without crystallisation. A typical cross section of a double clad uncoated GLSO fibre is shown in Fig. 2.

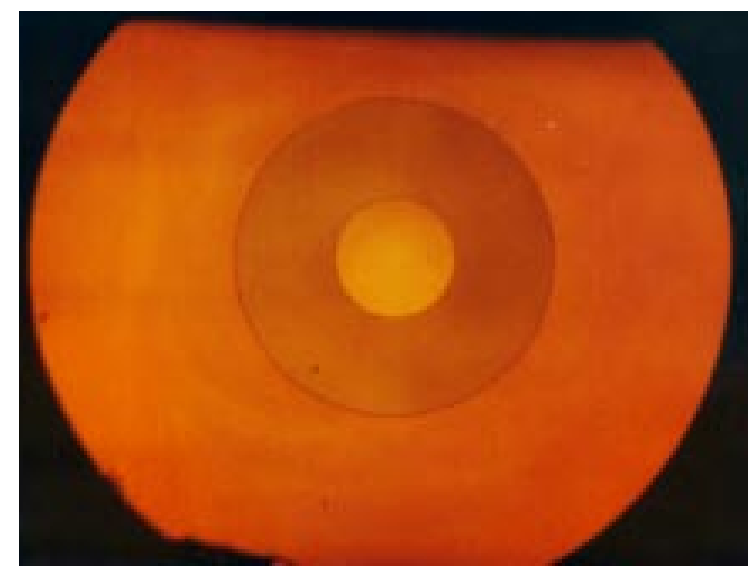

Fig. 2. Cross section of a double clad uncoated GLSO fibre. 
Minimum losses of about $4 \mathrm{dBm}^{-1}$ at $4 \mu \mathrm{m}$ have been achieved in unclad GLSO fibres. The attenuation spectrum is largely flat over a wide wavelength range. The loss in the $1-3 \mu \mathrm{m}$ region of the spectrum has a complex dependence on wavelength that is a combination of absorptions from transition metal impurities and scattering effects. Transition metal absorptions in GLS based glasses have been measured by intentionally doping the glass with transistion metals, to identify individual contributions of transition metal ions to the materials loss. In the $3-5 \mu \mathrm{m}$ region the absorption comes primarily from scattering from inclusions, crystallites and bubbles, and from $\mathrm{OH}^{-}$ impurity introduced by the $\mathrm{La}_{2} \mathrm{O}_{3}$. It is anticipated that purification of the starting materials will improve the loss considerably by removing the absorptions from transition metals, and also reducing the number of nucleation sites for crystallization, and hence the scattering loss. [2]

These glasses have several advantages over other glass materials as rare-earth hosts. The solubility of rare-earth ions is extremely high due to the presence of lanthanum as a glass former, and the emission cross sections of rare-earth levels are enhanced by the high refractive index $(n=2.4$ for GLS, 2.2 for GLSO). Combined with the low phonon energy, this potentially gives access to MIR transitions for lasers. We have chosen the erbium ion as an example to show the effect of the glass host on the spectroscopy of the incorporated rare-earth ions.

\section{Mid-infrared spectroscopy of erbium doped GLS glasses and fibres}

The trivalent erbium ion $\left(\mathrm{Er}^{3+}\right)$ is probably the most widely studied rare-earth dopant in glass fibres due to the success of the EDFA (Erbium Doped Fibre Amplifier) in silica based glasses. [3] EDFA's make use of the $1.55 \mu \mathrm{m}$ transition from the ${ }^{4} \mathrm{I}_{13 / 2}$ level to the ground state (Fig. 3). In silica glasses the ${ }^{4} \mathrm{I}_{13 / 2}$ level is the only metastable level with a typical lifetime of $10 \mathrm{~ms}$ whereas all the higher lying levels show lifetimes shorter than $10 \mu \mathrm{s}$. The high phonon energy of the glass host causes large non-radiative decay rates for levels with small energy gaps to the next lower level and quenches emission from these levels. Changing the host material from silica to a material with lower phonon energies considerably changes the spectroscopic properties of the embedded $\mathrm{Er}^{3+}$ ions.

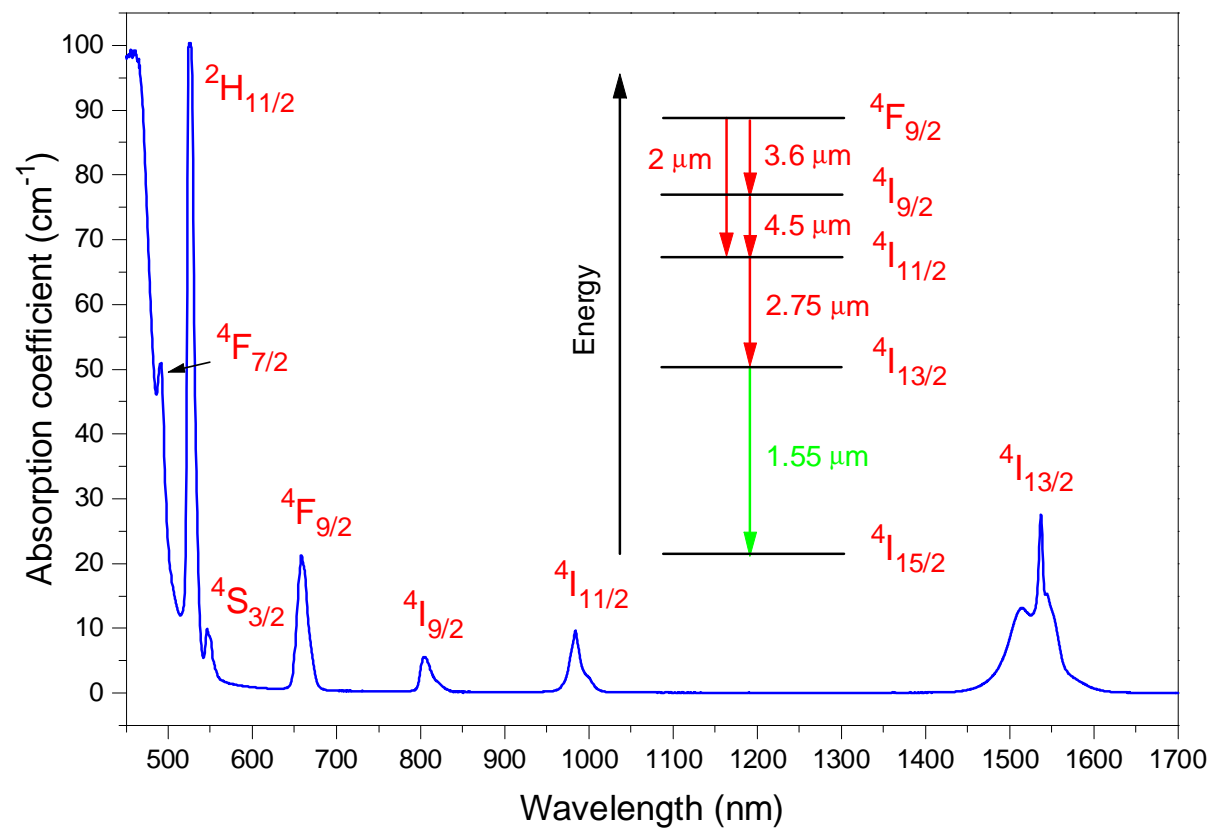

Fig. 3. Absorption spectrum of $9.7 \mathrm{~mol} \% \mathrm{Er}^{3+}$ doped GLS glass and $\mathrm{Er}^{3+}$ energy levels indicating the infrared transitions 
For spectroscopic measurements the $\mathrm{Er}^{3+}$ ions were excited at $660 \mathrm{~nm}\left({ }^{4} \mathrm{~F}_{9 / 2}\right)$ with a DCM dye laser and at $810 \mathrm{~nm}\left({ }^{4} \mathrm{I}_{9 / 2}\right)$ with a Ti:sapphire laser. Fluorescence spectra were measured with a $300 \mathrm{~mm}$ monochromator and a liquid nitrogen cooled InSb detector. The fluorescence was collected from the end of the fibres and from the side of the bulk sample, both doped with $1.57 \mathrm{~mol} \% \mathrm{Er}^{3+}$. Spectra were corrected for system response and atmospheric gas absorptions using a black body source. Fig. 4 shows four infrared $\mathrm{Er}^{3+}$ emission bands at 2.0, 2.75, 3.6, and $4.5 \mu \mathrm{m}$. The inhomogeneously broadened emission line shapes, typical for glass hosts, offer the possibility of wide wavelengths tunability. None of these would be observed in silica glasses or other high phonon energy host materials.
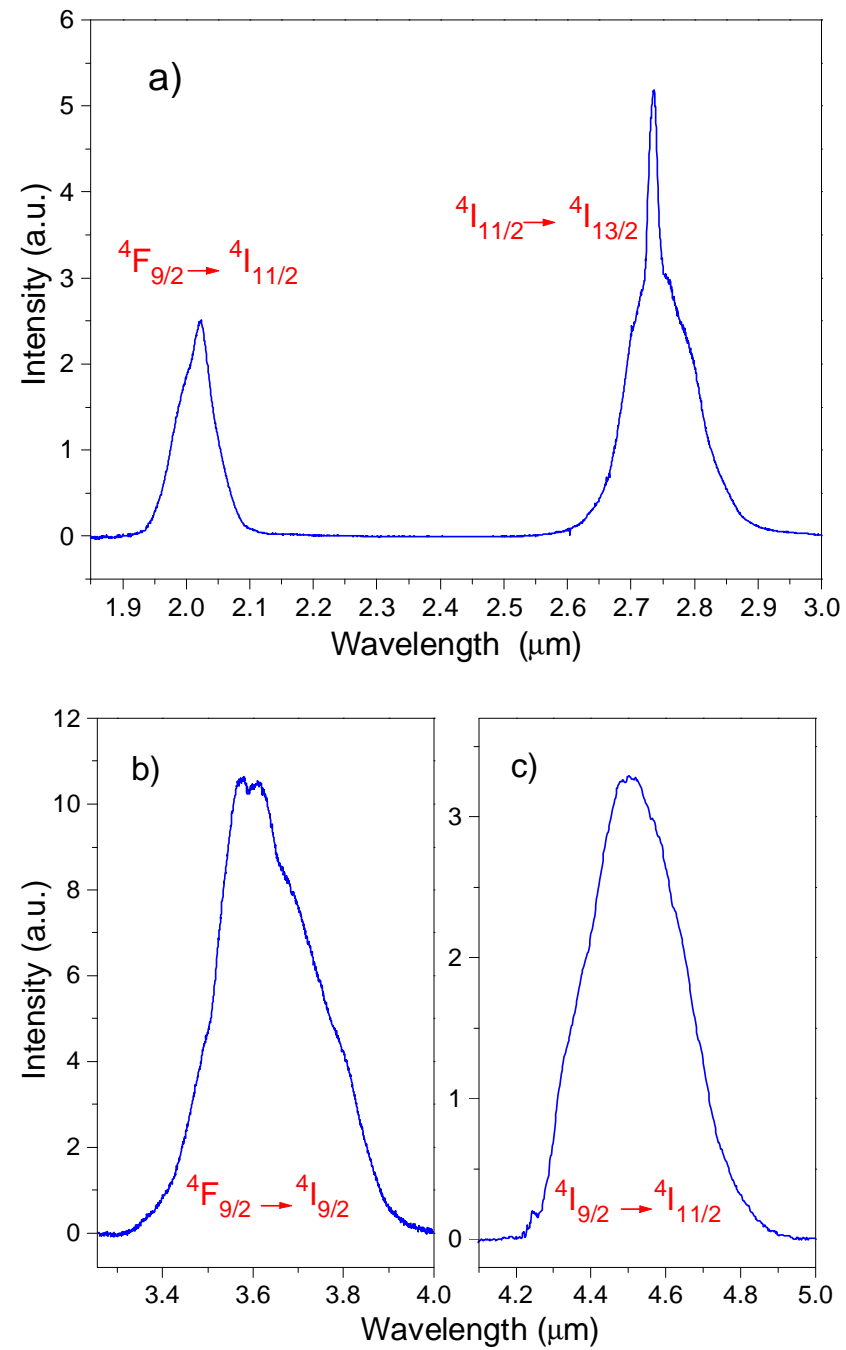

Fig. 4. Fluorescence spectra of $1.57 \mathrm{~mol} \% \mathrm{Er}^{3+}$ doped GLS glasses and fibres

a) $2.0 \mu \mathrm{m}$ and $2.75 \mu \mathrm{m}$ emission from $3.9 \mathrm{~cm}$ of $270 \mu \mathrm{m}$ diameter fibre pumped with $60 \mathrm{~mW}$ at $660 \mathrm{~nm}$

b) $3.6 \mu \mathrm{m}$ emission from $8.6 \mathrm{~cm}$ of $270 \mu \mathrm{m}$ diameter fibre pumped with $70 \mathrm{~mW}$ at $660 \mathrm{~nm}$

c) $4.5 \mu \mathrm{m}$ emission from a bulk glass sample pumped with $570 \mathrm{~mW}$ at $810 \mathrm{~nm}$

Judd-Ofelt calculations and lifetime measurements for $\mathrm{Er}^{3+}$ doped GLS glass are given in Ref. 4. We used the given electric and magnetic dipole contributions, $A_{\text {ed }}$ and $A_{\text {md }}$, of the radiative transition rate $\mathrm{A}=\mathrm{A}_{\mathrm{ed}}+\mathrm{A}_{\mathrm{md}}$ and the radiative and measured lifetimes $\tau_{\mathrm{r}}$ and $\tau_{\mathrm{m}}$ to calculate the branching ratios $\beta=\mathrm{A} \times \tau_{\mathrm{r}}$ and the quantum efficiencies $\eta=\beta \times \tau_{\mathrm{m}} / \tau_{\mathrm{r}}$ for the above transitions (Table 1). The emission cross sections $\sigma_{\mathrm{em}}$ were calculated from the 
radiative rates $A$ and the measured intensity spectra $I(\lambda)$ using the Füchtbauer-Ladenburg equation as given in Ref. 5:

$$
\sigma_{e m}(\lambda)=\frac{A \lambda^{5} I(\lambda)}{8 \pi n^{2} c \int \lambda I(\lambda) d \lambda}
$$

The refractive index $\mathrm{n}$ is 2.36 for all four transitions. Table 1 summarises the radiative properties of the four infrared transitions.

Table 1. Radiative properties of mid-infrared transitions in $\mathrm{Er}^{3+}$ doped GLS glass ( $\mathrm{A}_{\mathrm{ed}}, \mathrm{A}_{\mathrm{md}}, \tau_{\mathrm{r}}$, and $\tau_{\mathrm{m}}$ from Ref. 4$)$

\begin{tabular}{ccccccccc}
\hline Transition & $\lambda(\mu \mathrm{m})$ & $\mathrm{A}_{\mathrm{ed}}\left(\mathrm{s}^{-1}\right)$ & $\mathrm{A}_{\mathrm{md}}\left(\mathrm{s}^{-1}\right)$ & $\beta(\%)$ & $\tau_{\mathrm{r}}(\mu \mathrm{s})$ & $\tau_{\mathrm{m}}(\mu \mathrm{s})$ & $\eta(\%)$ & $\sigma_{\mathrm{em}}\left(10^{-20} \mathrm{~cm}^{2}\right)$ \\
\hline${ }^{4} \mathrm{~F}_{9 / 2} \rightarrow{ }^{4} \mathrm{I}_{11 / 2}$ & 2.02 & 249 & 18 & 3.7 & 140 & 100 & 2.6 & 1.52 \\
${ }^{4} \mathrm{~F}_{9 / 2} \rightarrow{ }^{4} \mathrm{I}_{9 / 2}$ & 3.62 & 21 & 8 & 0.4 & 140 & 100 & 0.3 & 0.43 \\
${ }^{4} \mathrm{I}_{9 / 2} \rightarrow{ }^{4} \mathrm{I}_{11 / 2}$ & 4.53 & 4 & 4 & 1.0 & 1200 & 590 & 0.5 & 0.25 \\
${ }^{4} \mathrm{I}_{11 / 2} \rightarrow{ }^{4} \mathrm{I}_{13 / 2}$ & 2.74 & 70 & 26 & 14.7 & 1530 & 1230 & 11.8 & 1.10 \\
\hline
\end{tabular}

The values in the second and third rows are in good agreement with the results reported recently by L.B. Shaw et. al who obtained branching ratios of $1 \%$ both for the $3.6 \mu \mathrm{m}$ and $4.5 \mu \mathrm{m}$ transitions with measured lifetimes of $100 \mu$ s and $500 \mu$ s and cross sections of $0.45 \times 10^{-20} \mathrm{~cm}^{2}$ and $0.25 \times 10^{-20} \mathrm{~cm}^{2}$, respectively, in an $\mathrm{Er}^{3+}$ doped barium indium gallium germanium sulphide glass. [6]

The measured lifetimes for the $0.0158 \mathrm{~mol} \% \mathrm{Er}^{3+}$ doped samples from Ref. 4 do not correspond to the size of the energy gaps to the next lower lying level, i.e. the lifetime of the $\quad{ }^{4} \mathrm{I}_{9 / 2}$ level is longer than the lifetime of the ${ }^{4} \mathrm{~F}_{9 / 2}$ level although the energy gap is smaller. This clearly indicates that the decay rates are dominated by the radiative decay and that non-radiative rates are very low in GLS glasses.

Nevertheless, quantum efficiencies are relatively low due to the low branching ratios, i.e. strong competing visible and near-infrared transitions from the same levels. However, the

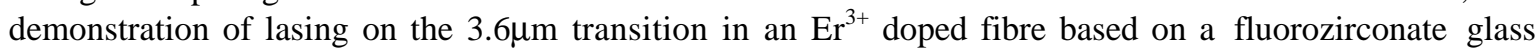
with less favourable spectroscopic properties (lower radiative rates and higher non-radiative rates) shows the potential for mid-infrared fibre lasers based on GLS glass. [7] A first step towards the realisation of these devices was the recent demonstration of the first rare-earth doped chalcogenide glass fibre laser in a $\mathrm{Nd}^{3+}$ doped GLS glass fibre at $1.08 \mu \mathrm{m}$. [8]

Radiation at $2 \mu \mathrm{m}$ finds application in LIDAR systems, radiation at $2.75 \mu \mathrm{m}$ coincides with a strong water absorption in tissue and is used for medical applications, the $3.6 \mu \mathrm{m}$ transition could be useful for $\mathrm{H}_{2} \mathrm{~S}, \mathrm{NO}$, and $\mathrm{SO}_{2}$ sensing or remote sensing depending on the wavelengths it is tuned to, and the $4.5 \mu \mathrm{m}$ transition could find use as $\mathrm{CO}$ and $\mathrm{O}_{3}$ gas sensors when tuned to $4.7 \mu \mathrm{m}$.

In conclusion we present the fabrication and spectroscopy of $\mathrm{Er}^{3+}$ doped gallium lanthanum sulphide based glasses and fibres showing the suitability of this glass host for rare-earth doped midinfrared fibre lasers.

\section{Acknowledgments}

Starting materials were supplied by Merck Ltd, Poole, UK. This work was in part carried out under the LINK Photonics project LONGWAVE with support from the DTI and the EPSRC.

1. D. W. Hewak, R. C. Moore, T. Schweizer, J. Wang, B. Samson, W. S. Brocklesby, D. N. Payne, E. J. Tarbox, "Gallium lanthanum sulphide optical fibre for active and passive applications," Electron. Lett. 32, 384-385 (1996)

2. J. S. Sanghera, V. Q. Nguyen, P. C. Pureza, R. E. Miklos, F. H. Kung, I. D. Aggarwal, "Fabrication of long lengths of low-loss IR transmitting $\mathrm{As}_{40} \mathrm{~S}_{(60-\mathrm{x})} \mathrm{Se}_{\mathrm{x}}$ glass fibre," J. Lightwave Technol. 14, 743-748 (1996)

3. P. Urquhart, "Review of rare earth doped fibre lasers and amplifiers," IEE Proc.-J: Optoelectron. 135, Pt. J, No. 6, $385-407$ (1988)

4. C.C. Ye, D.W. Hewak, M. Hempstead, B.N. Samson, and D.N. Payne, "Spectral properties of Er3+-doped gallium lanthanum sulphide glass," J. Non-Cryst. Solids 208, 56-63 (1996)

5. B. F. Aull, and H. P. Jenssen, "Vibronic interactions in Nd:YAG resulting in nonreciprocity of absorption and stimulated emission cross sections," IEEE J. Quantum Elect. QE-18, 925-930 (1982)

6. L.B. Shaw, D. Schaafsma, J. Moon, B. Harbison, J. Sanghera, I. Aggarwal, "Evaluation of the IR transitions in rare-earth-doped chalcogenide glasses," in Conference on Lasers and Electrooptics, Volume 11 of 1997 OSA Technical Digest Series (Optical Society of America, Washington, DC, 1997), p. 255.

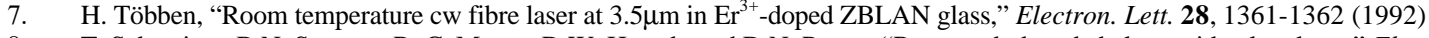

8. T. Schweizer, B.N. Samson, R. C. Moore, D.W. Hewak, and D.N. Payne, "Rare-earth doped chalcogenide glass laser," Electron. Lett. 33, 414-416 (1997) 
